\title{
Hybrid treatment of an unusual traumatic aortic arch rupture with pseudoaneurysm: a case report
}

\author{
Shoujun Tang' ${ }^{1}$ Shengjie Tang ${ }^{1}$, Li Yu², Yongheng Zhang ${ }^{1}$ and Haining Zhou ${ }^{1 *}$ (D)
}

\begin{abstract}
Background: Traumatic aortic arch rupture with pseudoaneurysm is a rare high fatal injury. Thoracic endovascular aortic repair(TEVAR) is widely used in the treatment of aortic diseases. However a unified traumatic aortic injury risk stratification tool still lacks. The patients' disease assessment and operation method still rely on the doctors' subjective anticipation of disease and surgical results.

Case presentation: A 31-year-old male presented with chest pain and hoarseness of recent onset. Imageological examination showed aortic rupture with mediastinal pseudoaneurysm in Zone 1. Debranching + TEVAR hybrid operation was considered: Using artificial blood vessels rebuild the arch branches, the covered stent was placed in aortic arch for endovascular repair via femoral artery. The follow-up was good in postoperative $7 d, 1$ year and $>2$ years.

Conclusion: The traumatic aortic arch rupture with pseudoaneurysm treated by Debranching+TEVAR hybrid operation is feasible. The short-term and medium-term follow-up results are satisfactory. For traumatic aortic arch injury, hybrid operation is recommended to reduce the risk of cardiopulmonary bypass and deep hypothermia circulatory arrest under no open operation conditions or emergency situation.
\end{abstract}

Keywords: Hybrid treatment, Traumatic, Pseudoaneurysm, Thoracic endovascular aortic repair (TEVAR)

\section{Background}

Blunt traumatic aortic injury (BTAI) is derived from the blunt injury due to decelerated impact. It is common in traffic accident and falling. The annual incidence rate is estimated $20-30 /$ million in the United States, accounting for $0.3 \%$ of traumatic events [1]. In 1958, Parmley reported that the pre-hospital mortality of BTAI was $85 \%$. With the increase of the pre-hospital first-aid level, the pre-hospital mortality of these patients was decreased to $54-80 \%$. The mortality was $50 \%$ after admission for $24 \mathrm{~h}$ and continued to be the second fatal cause of traumatic patients [2]. The very low morbidity and high mortality make patients difficultly survive and be admitted in hospital.

\footnotetext{
*Correspondence: zhouhaining@aliyun.com

${ }^{1}$ Cardiovascular Center, Suining Central Hospital, No.127, West Desheng

Road, Chuanshan District, Suining 629000, Sichuan, China

Full list of author information is available at the end of the article
}

\section{Case presentation}

A 31-year-old male presented with chest pain and hoarseness of recent onset. The patient had suffered a traffic accident 3 months before admission, resulting with femur, radius, ribs and sternum fractures; and had undergone internal fixation of the femur and radius. The physical examination was normal. Computed tomography (CT) showed a ruptured aortic arch with pseudoaneurysm. The crevasse measured $20 \mathrm{~mm}$ and was located in the posteroinferior aspect of the aortic arch, involving the posterior wall of the innominate artery and the origin of left common carotid artery. The pseudoaneurysm of $40 \mathrm{~mm} \times 48 \mathrm{~mm} \times 30 \mathrm{~mm}$ was located in the upper mediastinum. The distance from the junction of aortic sinus and ascending aorta to the rupture was 7.9 $\mathrm{cm}$ (Figs. $1 \mathrm{a}-\mathrm{c}$ ). The recent appearance of symptoms suggested that the pseudoaneurysm had increased rapidly and compressed the left recurrent laryngeal nerve. In this case, to reduce the risk of cardiopulmonary bypass or cardiac arrest under deep hypothermia [3], a 

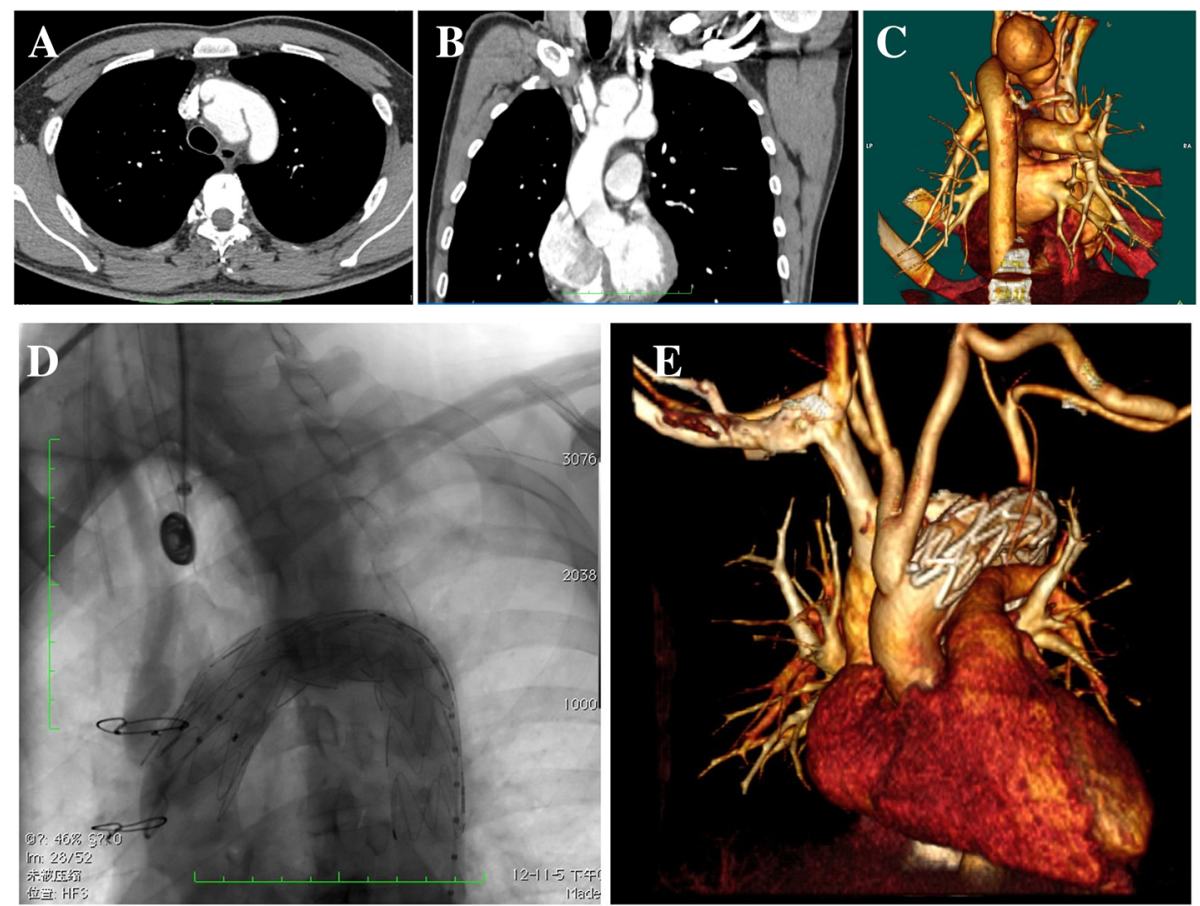

Fig. 1 (a-c) Preoperative $C T$; (d) Intraoperative angiography; (e) $C T$ angiography on the 7th postoperative day

hybrid operation was performed: debranching + thoracic endovascular aortic repair (TEVAR). The involved branches of the aortic arch were rebuilt with artificial vessels, and a covered stent was placed in the aortic arch for endovascular repair via femoral artery. A hybrid operation is safer, more feasible, and more comprehensive than other treatments for some high-risk patients. Thoracic and abdominal CT angiography performed on the 7th postoperative day showed that the contrast agent did not leak and that the reconstruction of blood vessels was smooth (Figs. $1 \mathrm{~d}$ and e). Recovery was uneventful.

\section{Discussion}

Blunt traumatic aortic injury is a rare high fatal decelerated injury. Its symptoms are obvious but signs are not obvious. The injury part is usually located in the aortic isthmus (54-65\%), rare in the ascending aorta at the proximal end of the innominate artery (10-20\%), aortic arch and descending aorta [4]. Once aortic aneurysm was missed or early expansion was not obvious, it was difficult to find and the accidental death much more likely occur. A few patients without symptoms were diagnosed by accident examination or chest and back pain, superior vena cava syndrome, hoarseness, dysphagia, cough, hemoptysis and other symptoms occurred. This kind of symptoms often suggested that the tumor body was increased and the disease was evolved. Once the diagnosis was confirmed, the timely surgical repair should be performed.
Endovascular repair is widely used in the treatment of aortic diseases. The Debranching endovascular repair achieved satisfactory short-term and mid-term results in aortic arch injury. The Hybrid operation mode dose not require cardiopulmonary bypass or deep hypothermia circulatory arrest, which provides a less invasive and treatment of injury of aortic arch [5].

The rupture position, anchoring region Debranching involvement and aortic arch curvature require to be carefully evaluated to prevent the "bird beaking" after stent implantation, and then to increase the endoleak rate even aortic rupture. The sequential clipping order of Debranching required to be evaluated, through CTA or MRA or digital subtraction angiography for the cerebral blood vessels. In this case, the patient was the left vertebral artery dominant. Debranchings were clipped and anastomosed from right to left. The left common carotid artery-branch vessels end-to-end anastomosis and left common carotid artery-left subclavian artery bypass anastomosis were performed on the neck incision and supraclavicular incision. After the left common carotid artery was clipped, the anastomosis of the two could be performed at the same time, so as to reduce the occlusion time of left common carotid artery. In order not to block the left vertebral artery, left subclavian artery anastomosis was performed in the distal end of initial left vertebral artery. The length of the proximal landing zone of the aortic arch was recommended for at least $20 \mathrm{~mm}$. The diameter of the stent 
was larger than $10-15 \%$ of the landing zone, so as to avoid the type I endoleak. After the left common carotid artery-left supraclavicular bypass was completed, the proximal end of the left clavicle artery was ligated or closed with a coil or plug via the left radial artery, to prevent type II endoleaks.

\section{Conclusions}

Traumatic pseudoaneurysm is a serious complication of local vascular injury. Hybrid debranching technique greatly reduces surgical trauma and provides satisfactory outcome and good function recovery. It is safer, more feasible, and more comprehensive than other treatments for some high-risk patients.

\section{Abbreviations}

BTAl: Blunt traumatic aortic injury; CT: computed tomography;

TEVAR: thoracic endovascular aortic repair

\section{Acknowledgements}

The authors are indebted to Dr. Ramon Rami-Porta, from the Department of Thoracic Surgery of Hospital Universitari Mutua Terrassa, in Terrassa, Spain, for his revision of the original manuscript and thoughtful suggestions.

\section{Funding}

None.

\section{Availability of data and materials}

All data generated or analysed during this study are included in the published article.

\section{Authors' contributions}

SJT, SGT and HNZ were involved in patient care. YHZ and LY were involved in manuscript preparation and revisions. All authors read and approved the final manuscript.

\section{Ethics approval and consent to participate}

The patient provided full consent for the publication of the data. The need for ethics approval was not required describing this case.

\section{Consent for publication}

Written informed consent was obtained from the patient for publication of this case report and any accompanying images. A copy of the written consent is available for review by the Editor-in-Chief of this journal.

\section{Competing interests}

The authors declare that they have no competing interests.

\section{Publisher's Note}

Springer Nature remains neutral with regard to jurisdictional claims in published maps and institutional affiliations.

\section{Author details}

${ }^{1}$ Cardiovascular Center, Suining Central Hospital, No.127, West Desheng Road, Chuanshan District, Suining 629000, Sichuan, China. ${ }^{2}$ Department of ECG, Suining Central Hospital, Suining 629000, China.

Received: 17 January 2019 Accepted: 1 April 2019 Published online: 08 April 2019

\section{References}

1. Fox N, Schwartz D, Salazar JH. Evaluation and management of blunt traumatic aortic injury. J Trauma Nurs. 2015;22:99-110.

2. Parmeley LF, Mattingly TW, Manion WC, Jahnke EJ. Nonpenetrating traumatic injury of the aorta. Circulation. 1958;17:1086-101.
3. Riesenman P, Tamaddon HS, Farber MA. Surgical bypass procedures to facilitate endovascular repair of aortic arch pathology. J Cardiovasc Surg. 2008;49:461-9.

4. Shackford SR, Dunne CE, Karmy-Jones R, et al. The evolution of care improves outcome in blunt thoracic aortic injury: a Western trauma association multicenter study. J Trauma Acute Care Surg. 2017;83:1006-13.

5. Demetriades D, Velmahos GC, Scalea TM, et al. Operative repair or endovascular stent graft in blunt traumatic thoracic aortic injuries: results of an American Association for the Surgery of Trauma multicenter study. J Trauma. 2008;64:561-70 discussion 570-1. 